Matsushima, $\mathrm{Y}$.

Osaka J. Math.

5 (1968), 215-222

\title{
AFFINE STRUCTURES ON COMPLEX MANIFOLDS
}

\author{
Yozô MATSUSHIMA
}

(Received May 9, 1968)

Let $M$ be a complex manifold of complex dimension $n$ and let $C=\left\{U_{i}, \phi_{i}\right\}_{i \in I}$ be the maximal atlas defining the complex structure on $M$. A subset $A=$ $\left\{U_{j}, \varphi_{j}\right\}_{j \in J}, J \subset I$, of $C$ is called an affine atlas of $M$, if $\varphi_{j k}=\varphi_{j} \circ \varphi_{k}^{-1}$ is a complex affine transformation of $\boldsymbol{C}^{n}$ whenever $U_{j} \cap U_{k} \neq \phi$. We can define the notion of a maximal affine atlas of $M$ and we say that each maximal affine atlas of $M$ defines a complex affine structure of the complex manifold $M$. We shall denote by $\boldsymbol{A}(M)$ the totality of complex affine structures on $M$.

The aim of this note is to study the structure of $\boldsymbol{A}(M)$ in the case where $M$ is compact and the complex structure of $M$ is homogeneous and we shall prove the following theorems.

Theorem 1. Let $M$ be a complex torus of complex dimension $n$. Then there exists a natural one-to-one correspondence between the set $\boldsymbol{A}(M)$ and the set of all commutative associative algebra structure over $\boldsymbol{C}$ in the complex vector space $\boldsymbol{C}^{\boldsymbol{n}}$. In particular $\boldsymbol{A}(M)$ is a complex affine variety.

More generally:

Theorem 2. Let $M$ be a connected compact complex manifold and let Aut $(M)$ be the group of all holomorphic transformations of $M$. Assume Aut $(M)$ is transitive on $M$. Then $\boldsymbol{A}(M)$ is a complex affine algebraic variety.

1. A) Let $M$ be complex manifold and let $I$ be the tensor of the almost complex structure associated with $M$. For each point $p \in M$, the value $I_{p}$ of $I$ at $p$ is an endomorphism of the tangent space $T_{p}(M)$ such that $I_{p}{ }^{2}=-1$. Let $T_{p}^{+}(M)$ (resp. $T_{x}^{-}(M)$ ) be the subspace of the complexified tangent space $T_{p}{ }^{C}(M)$ consisting of all $u$ such that $I_{p} u=i u\left(\right.$ resp. $\left.I_{p} u=-i u\right)$ with $i=\sqrt{-1}$. Then we have

$$
T_{p}{ }^{C}(M)=T_{p}^{+}(M) \oplus T_{p}^{-}(M) .
$$

If $\left\{z^{1}, z^{2}, \cdots, z^{n}\right\}$ is a system of complex local coordinates on an open set $U$, then $\left\{\left(\partial / \partial z^{i}\right)_{p}\right\}_{i=1, \cdots, n}$ and $\left\{\left(\partial / \partial \bar{z}^{i}\right)_{p}\right\}_{i=1, \cdots, n}$ are bases of $T_{p}^{+}(M)$ and $T_{p}^{-}(M)$ respectively at each point $p \in U$. The totality of complex tangent vectors belonging to $T_{p}^{+}(M)(p \in M)$ form a holomorphic vector bundle $T^{+}(M)$ over $M$. 
Let $X$ be a smooth vector field on $M$. Then we can write $X$ uniquely in the form

$$
X=X^{+}+X^{-}
$$

where $X^{+}(p) \in T_{p}^{+}(M)$ and $X^{-}(p) \in T_{p}^{-}(M)$ at each point $p \in M$ and $X^{-}(p)=$ $\overline{X^{+}(p)}$, where-denotes the conjugation of $T_{p}{ }^{C}(M)$.

A complex vector field $W$ on $M$ is, by definition, a smooth section of the vector bundle $T^{+}(M) \oplus T^{-}(M)$. Then we can write $W$ uniquely in the form $W=X+i Y$, where $X$ and $Y$ are smooth real vector fields on $M$. A complex vector field $W$ is called holomorphic if $W$ is a holomoprhic section of $T^{+}(M)$. A smooth real vector field $X$ is called holomorphic if $X^{+}$is holomorphic.

Let $\mathfrak{g}=\mathrm{g}(M)$ be the vector space of all holomorphic real vector fields on $M$. Then $\mathrm{g}$ is a complex Lie algebra and if $M$ is compact, $\mathfrak{g}$ is identified with the Lie algebra of the group Aut $(M)$ of holomorphic transformations of $M$.

In the following we denote by $\mathfrak{X}(M)$ the real vector space of all smooth vector fields on $M$. Then a complex vector field on $M$ is identified with an element of $\mathfrak{X}(M)^{C}$.

B) A linear connection $\nabla$ on $M$ is defined by a bilinear mapping $(X, Y) \rightarrow$ $\nabla_{X} Y$ of $\mathfrak{X}(M) \times \mathfrak{X}(M)$ into $\mathfrak{X}(M)$ satisfying the following conditions:

1) $\nabla_{f Y} X \neq f\left(\nabla_{Y} X\right)$

2) $\nabla_{Y} f X=f\left(\nabla_{Y} X\right)+Y f \cdot X$.

A linear connection $\nabla$ on $M$ is called a holomorphic linear connection if the following two conditions are satisfied:

a) $\nabla_{Y} I X=I\left(\nabla_{Y} X\right)$ for all $X, Y \in \mathfrak{X}(M)$;

b) if $X$ and $Y$ are holomorphic vector fields defined on an open set $O$ of $M$, then $\nabla_{Y} X$ is also holomorphic on $O$.

If $\nabla$ is a linear connection, we can extend $\nabla$ to a complex bilinear mapping of $\mathfrak{X}(M)^{c} \times \mathfrak{X}(M)^{C}$ into $\mathfrak{X}(M)^{C}$. Then the conditions a) and $\mathrm{b}$ ) are equivalent to the following two conditions $\left.\mathrm{a}^{\prime}\right)$ and $\left.\mathrm{b}^{\prime}\right)$.

$\left.\mathrm{a}^{\prime}\right) \quad\left(\nabla_{Y} X\right)^{+}=\nabla_{Y} X^{+}$;

$\left.\mathrm{b}^{\prime}\right)$ if $U$ and $W$ are complex holomorphic vector fields defined on an open set $O$, then $\nabla_{W} U$ is also holomorphic.

C) Let us consider a complex affine structure on $M$ defined by a maximal affine atlas $\{(O, \varphi)\}$. Let $\left\{z^{1}, \cdots, z^{n}\right\}$ be the local coordinates on $O$ defined by the chart $(O, \varphi)$. On each of these open sets $O$ we can define uniquely an linear connection $\nabla^{0}$ on $O$ by the conditions: $\nabla_{Z^{i}}^{0} Z^{j}=\nabla_{\bar{Z}}^{0} Z^{j}=0(i, j=1,2, \cdots, n)$, where $Z^{i}=\partial / \partial z^{i}$ and $\bar{Z}^{i}=\partial / \partial z^{i}$. Then there exists a unique linear connection $\nabla$ on $M$ such that the restriction of $\nabla$ on each $O$ coincides with $\nabla^{0}$. This affine connection $\nabla$ is holomorphic and locally flat, i.e. the torsion and the curvature of $\nabla$ are 0 . This means that 


$$
\begin{aligned}
& \nabla_{X} Y-\nabla_{Y} X=[X, Y] \\
& \nabla_{X}\left(\nabla_{Y} Z\right)-\nabla_{Y}\left(\nabla_{X} Z\right)=\nabla_{[X, Y]} Z
\end{aligned}
$$

for any $X, Y, Z \in \mathfrak{X}(M)$.

Thus to each affine structure on $M$ there corresponds a locally flat, holomorphic linear connection on $M$ and to the distinct affine structures there correspond distinct linear connections.

D) Let, conversely, $\nabla$ be any locally flat, holomorphic linear connection on $M$ and let $\tilde{M}$ be the universal covering manifold of $M$. Then there is defined uniquely a connection $\tilde{\nabla}$ on $\tilde{M}$ such that, for smooth vector fields $X$ and $Y$ on $M$, we have $\tilde{\nabla}_{Y^{*}} X^{*}=\left(\nabla_{Y} X\right)^{*}$, where, for each vector field $X$ on $M, X^{*}$ denotes the lift of $X . \quad \tilde{\nabla}$ is also locally flat and holomorphic. Let $\mathfrak{H}=\{P\}$ be the complex vector space of all parallel complex holomorphic vector fields on $\tilde{M}$. Then the map $P \rightarrow P(\tilde{a})(\tilde{a} \in \tilde{M})$ is a bijection of $\mathfrak{\beta}$ onto $T_{\tilde{a}}^{+}(M)$. In particular $\operatorname{dim}_{C} \mathfrak{P}=n=\operatorname{dim}_{C} M$. $\mathfrak{P}$ form an abelian Lie algebra, because $\left[P, P^{\prime}\right]=$ $\tilde{\nabla}_{P} P^{\prime}-\tilde{\nabla}_{P^{\prime}} P=0$.

Let $\Gamma$ be the fundamental group of $M$. Then $\Gamma$ acts from the right on $\tilde{M}$ and the action of each element of $\Gamma$ is holomorphic and affine.

Fix a point $a \in M$ and let $\tilde{a}$ be a point of $\tilde{M}$ such that $\pi(\tilde{a})=a, \pi$ denoting the projection of $\tilde{M}$ onto. $M$. For each vector $y \in T_{a}^{+}(M)$, there exists one and only one $P \in \Re$ such that $d \pi \cdot P(\tilde{a})=y$. We denote this vector field $P$ by $P_{y}$.

Let $\gamma \in \Gamma$. Then $\gamma$ is a holomorphic affine transformation and hence $d \gamma \cdot \mathfrak{P} \subset$. Put

$$
d \gamma \cdot P_{y}=P_{f(\gamma) y}
$$

Then $\gamma \rightarrow f(\gamma)$ is a representation of the group $\Gamma$ in the vector space $T_{a}^{+}(M)$.

Now let $\left\{P_{1}, \cdots, P_{n}\right\}\left(n=\operatorname{dim}_{C} M\right)$ be a basis of the complex vector space $\mathfrak{B}$. Then we can define $n$ holomorphic 1 -forms $\left\{\omega^{1}, \cdots, \omega^{n}\right\}$ on $\tilde{M}$ by the condition

$$
\omega^{i}\left(P_{j}\right)=\delta_{j}^{i} \quad(i, j=1, \cdots, n) .
$$

These 1 -forms are closed. There exists a basis $\left\{y_{1}, \cdots, y_{n}\right\}$ of $T_{a}^{+}(M)$ such that $P_{i}=P_{y_{i}}(i=1,2, \cdots$,$) . We can define a T_{a}^{+}(M)$-valued holomorphic 1 -form $\theta$ on $\tilde{M}$ by

$$
\theta=\sum_{i=1}^{n} \omega^{i} y_{i}
$$

Then we have:

$$
\theta(P)=d \pi \cdot P(\tilde{a}), \quad P \in \mathfrak{F},
$$

and

$$
d \theta=0
$$


Moreover,

$$
(d \gamma)^{*} \theta=f(\gamma) \cdot \theta, \quad \gamma \in \Gamma .
$$

In fact, let $P \in \mathfrak{F}$. Then there exists $y \in T_{a}^{+}(M)$ such that $P=P_{y}$. Then

$$
((d \gamma) * \theta)(P)=\theta\left(d \gamma P_{y}\right)=\theta\left(P_{f(\gamma) y}\right)=f(\gamma) \cdot y \quad \text { by }(2) .
$$

On the other hand, by (2) $y=\theta(P)$ and hence $\left((d \gamma)^{*} \cdot \theta\right)(P)=f(\gamma) \cdot \theta(P)$ and this proves the equality (4). For any $\tilde{x} \in \tilde{M}$, let

$$
\varphi(\tilde{x})=\int_{\tilde{a}}^{\tilde{x}} \theta .
$$

Then $\phi$ is a holomorphic map of $\tilde{M}$ into $T_{a}^{+}(M)$. Put

$$
q(\gamma)=\varphi(\gamma \tilde{a})
$$

for all $\gamma \in \Gamma$. We have then

$$
\varphi(\gamma \tilde{x})=f(\gamma) \varphi(\tilde{x})+q(\gamma) .
$$

In particular for $\tilde{x}=\sigma \tilde{a}(\sigma \in \Gamma)$, we have

$$
q(\gamma \sigma)=f(\gamma) q(\sigma)+q(\gamma) .
$$

This shows that $q$ is a 1-cocycle of the group $\Gamma$ and that, if we denote $a(\gamma)$ the complex affine transformation $x \rightarrow f(\gamma) x+q(\gamma)$ of $T_{a}^{+}(M)$, then $\gamma \rightarrow a(\gamma)$ is a homomorphism of $\Gamma$ into the group of complex affine transformations of $T_{a}^{+}(M)$. Moreover (7) shows that

$$
\varphi(\gamma \tilde{x})=a(\gamma) \varphi(\tilde{x}) .
$$

By the definition of $\varphi$, we have

$$
(d \varphi)(\tilde{x})=\theta(\tilde{x})
$$

and $\theta(\tilde{x}): T_{\tilde{x}}^{+}(M) \rightarrow T_{a}^{+}(M)$ is bijective. Therefore $\varphi$ is a holomorphic immersion of $\tilde{M}$ into the $n$ dimensional complex vector space $T_{a}^{+}(M)$ which satisfies $\left(7^{\prime}\right)^{*}$. Let $U$ be an open subset of $M$ evenly covered by $\pi$ such that each connected component of $\pi^{-1}(U)$ is mapped bijectively by $\varphi$ onto an open set in $\boldsymbol{C}^{n}=T_{a}^{+}(M)$. Let $\widetilde{U}$ be any one of the connected components of $\pi^{-1}(U)$ and let $\psi$ be the holomorphic bijective map of $U$ onto $\varphi(\widetilde{U})$ defined by $\psi=\varphi \circ(\pi \mid \widetilde{U})^{-1}$. Then it is easy to check that $\{(U, \psi)\}$ defines a complex affine structure on $M$ and that the locally flat holomorphic linear connection associated with this complex affine structure coincides with $\nabla$.

* The mapping $\varphi$ is the "development" of $\tilde{M}$ in $\boldsymbol{C}^{n}$, The present way of defining the development $\varphi$ is due to J.L. Koșzul, 
Thus there is a one-to-one correspondence between the set $\boldsymbol{A}(M)$ of all complex affine structures on a complex manifold $M$ and the set of all locally flat, holomorphic linear connections on $M$.

2. In the following we shall denote by $\boldsymbol{A}(M)$ the set of all locally flat, holomorphic linear connections on $M$. We denote by $g$ the complex Lie algebra of all holomorphic vector fields on $M$. From now on we assume that $M$ is compact. Then $\mathrm{g}$ is identified with the Lie algebra of the group Aut $(M)$.

Now let $\nabla \in \boldsymbol{A}(M)$. Then the map $(X, Y) \rightarrow-\nabla_{Y} X$ is a bilinear map of $\mathfrak{g} \times \mathfrak{g}$ into $\mathfrak{g}$. Let

$$
X \cdot Y=-\nabla_{Y} X .
$$

Then this multiplication in $g$ defines an algebra structure on the complex vector space $g$ and we denote this algebra by $\mathfrak{g}(\nabla)$.

Definition. Let $A$ be an algebra over a field $k$ and set $[x, y, z]=$ $x(y z)-(x y) z$ and call it the associator of $x, y$ and $z$. We call $A$ a pre-Lie algebra, if the relation

$$
[x, y, z]=[x, z, y]
$$

holds for any $x, y$ and $z$ in $A$.

For example, an associative algebra $A$ is a pre-Lie algebra. Let $A$ be a pre-Lie algebra and set

$$
[x, y]=x y-y x
$$

for $x, y \in A$. Then we can show and that the bracket product $[x, y]$ defines a Lie algebra. We call this Lie algebra the Lie algebra associated with $A$.

Remark. The notion of pre-Lie algebras has been introduced by $M$. Gerstenhaber in connection with the deformation of algebras. See [1] and [3].

Definition. Let $\mathfrak{g}$ be a Lie algebra over a field $k$ and $A$ a pre-Lie algebra over $k$. We call $A$ pre-Lie algebra over $\mathrm{g}$ if the associated Lie algebra of $A$ is $\mathrm{g}$.

Lemma 1. Let $\mathrm{g}$ be the algebra of all holomorphic vector fields on a compact complex manifold $M$ and let $\nabla$ be a locally flat holomorphic linear connection on $M$. Then the algebra $\mathrm{g}(\nabla)$ is a pre-Lie algebra over $\mathrm{g}$.

This lemma follows easily from the definition of the multiplication in $g(\nabla)$ and from the fact that the torsion and the curvature of $\nabla$ are 0 .

Let us denote by $\boldsymbol{A}(\mathfrak{g})$ the set of all pre-Lie algebra structures over $\mathfrak{g}$. Then the map $\nabla \rightarrow \mathrm{g}(\nabla)$ defines a map of $\boldsymbol{A}(\boldsymbol{M})$ into $\boldsymbol{A}(\mathrm{g})$.

Assume now that $M$ is homogeneous. Then for any $p \in M$, the tangent vectors $X(p)(X \in \mathrm{g})$ span the tangent space $T_{p}(M)$. Then we see easily that 
the map of $\boldsymbol{A}(M)$ into $\boldsymbol{A}(\mathfrak{g})$ is injective.

Now let

$$
\mathfrak{g}_{p}=\{Y \in \mathfrak{g} \mid Y(p)=0\}, \quad p \in M .
$$

If $Y \in \mathfrak{g}_{p}$, then $\left(\nabla_{Y} X\right)(p)=\nabla_{Y(p)} X=0$ and hence $\mathfrak{g}_{p}$ is a left ideal of $\mathfrak{g}(\nabla)$.

Conversely we have the following lemma.

Lemma 2. Let $M$ be a compact homogeneous complex manifold and let $\mathrm{g}$ be the Lie algebra of all holomorphic vector fields on $M$. Let $A$ be a pre-Lie algebra over $\mathrm{g}$ such that $\mathrm{g}_{p}$ is a left ideal of $A$ for every $p \in M$. Then there exists a locally flat holomorphic linear connection $\nabla$ on $M$ such that $A=\mathfrak{g}(\nabla)$.

Proof. Let $p \in M$ and $u \in T_{p}(M)$. Then there exists a $Y \in \mathrm{g}$ such that $Y(p)=u$. For any $X \in \mathrm{g}$ define $\nabla_{u} X \in T_{p}(M)$ by putting

$$
\nabla_{u} X=-(X \cdot Y)(p) \text {. }
$$

This definition does not depend on the choice of $Y \in \mathfrak{g}$ such that $Y(p)=u$, because $\mathrm{g}_{p}$ is a left ideal of $A$. For any vector field $Y$ and any $X \in \mathfrak{g}, \nabla_{Y} X$ will denote the vector field on $M$ such that

$$
\left(\nabla_{Y} X\right)(p)=\nabla_{Y(p)} X .
$$

Then the following equalities hold:

1) $\nabla_{f y} X=f \nabla_{Y} X$, where $f$ is a smooth function on $M$;

2) $\nabla_{Y+Y^{\prime}} X=\nabla_{Y} X+\nabla_{Y^{\prime}} X ; \nabla_{Y}\left(X+X^{\prime}\right)=\nabla_{Y} X+\nabla_{Y} X^{\prime}$,

where $Y$ and $Y^{\prime}$ are smooth vector fields on $M$ and $X, X^{\prime} \in \mathrm{g}$;

3) $\nabla_{y} X$ is a smooth vector field; in fact, let $p \in M$. Then in a neighborhood $U$ of $p, Y$ is written uniquely in the form $Y=f^{1} Y_{1}+\cdots+f^{n} Y_{n}$, where $Y_{1}, \cdots, Y_{n}$ are in $g$ and $f^{1}, \cdots, f^{n}$ are smooth functions on $U$. At each point $q \in U$, we have $\left(\nabla_{Y} X\right)(q)=\sum_{i=1}^{n} f^{i}(q)\left(\nabla_{Y_{i}} X\right)(q)=-\sum_{i=1}^{n} f^{i}(q)\left(X \cdot Y_{i}\right)(q)$ and hence $\nabla_{Y} X$ is smooth on $U$.

Next let $Y$ be a smooth vector field and $u \in T_{p}(M)$. Define $\nabla_{u} Y \in T_{p}(M)$ by

$$
\nabla_{u} Y=\nabla_{Y(p)} X+[X, Y](p),
$$

where $X$ is a vector field in $\mathfrak{g}$ such that $u=X(p)$. We have to show that this definition is consistent. It suffices to show that

$$
\nabla_{Y(p)} X+[X, Y](p)=0
$$

whenever $X(p)=0$ and $X \in \mathfrak{g}$. To see this let $Y=f^{1} Y_{1}+\cdots+f^{n} Y_{n}$ in a neighborhood $U$ of $p$, where $Y_{1}, \cdots, Y_{n} \in \mathrm{g}$. Then

$$
\nabla_{Y(p)} X=\sum_{i=1}^{n} f^{i}(p) \nabla_{Y_{i}(p)} X \quad \text { and } \quad[X, Y]=\sum_{i=1}^{n} f^{i}\left[X, Y_{i}\right]+\sum_{i=1}^{n} X f^{i} \cdot Y_{i}
$$


on $U$. Since $X(p)=0$, we have $\left(X f^{i}\right)(p)=0$ and, since $X$ and $Y_{i}$ are in $\mathfrak{g},\left[X, Y_{i}\right]=X \cdot Y_{i}-Y_{i} \cdot X$. Therefore $\left[X, Y_{i}\right](p)=\left(X \cdot Y_{i}\right)(p)-\left(Y_{i} \cdot X\right)(p)=$ $-\nabla_{Y_{i}(p)} X+\nabla_{X(p)} Y_{i}=-\nabla_{Y_{i}(p)} X$ and hence $\nabla_{Y(p)} X+[X, Y](p)=\sum_{i=1}^{n} f^{i}(p)$ $\nabla_{Y_{i}(p)} X-\sum_{i=1}^{n} f^{i}(p) \nabla_{Y_{i}(p)} X=0$. Thus we have defined the tangent vector $\nabla_{u} Y$ for any $u \in T_{p}(M)$ and any smooth vector field $Y$. The following conditions hold:

i) $\nabla_{u}\left(Y+Y^{\prime}\right)=\nabla_{u} Y+\nabla_{u} Y^{\prime}$

ii) $\nabla_{u+v} Y=\nabla_{u} Y+\nabla_{v} Y ; \nabla_{\lambda u} Y=\lambda \nabla_{u} Y \quad(\lambda \in \boldsymbol{R})$;

iii) $\nabla_{u}(f Y)=f(p) \nabla_{u} Y+u f \cdot Y$, where $f$ is a smooth function.

Thus we have defined a linear connection $\nabla$ on $M$ and it is easily seen that the torsion of $\nabla$ is 0 and that $\nabla_{Y} X=-X \cdot Y$ for $X, Y \in \mathrm{g}$. Then $\nabla_{X}\left(\nabla_{Y} Z\right)-$ $\nabla_{Y}\left(\nabla_{X} Z\right)-\nabla_{[X, Y]} Z=[Z, X, Y]-[Z, Y, X]=0$ for $X, Y, Z \in \mathrm{g} . \quad$ It follows then that the curvature of $\nabla$ is 0 . Moreover it is easily seen that $\nabla$ is holomorphic and $\mathrm{g}(\nabla)=A$ and the lemma is proved.

Assume now that $g_{p}=\{0\}$ for all $p \in M$. This is the case if and only if $M$ is of the form $M=G / D$, where $G$ is a complex Lie group and $D$ is a discrete subgroup of $G$. In this case, the map $\nabla \rightarrow \mathfrak{g}(\nabla)$ establishes a one-to-one correspondence between the set of all complex affine structures on $M$ and the set $\boldsymbol{A}(\mathfrak{g})$ of all pre-Lie algebra structures over $\mathrm{g}$. In particular, this holds for a complex torus $M$. In this case the Lie algebra $g$ is abelian and Theorem 1 follows from the following lemma and from what we have proved so far.

Lemma 3. Let $A$ be a pre-Lie algebra over a Lie algebra $\mathfrak{g}$. Assume $\mathfrak{g}$ is abelian. Then $A$ is a commutative associative algebra.

In fact, $x y-y x=[x, y]=0$ and hence $x y=y x$ for $x, y \in A$. Moreover, $x(y z)-(x y) z=x(z y)-(x z) y$ and $y z=z y$ and hence $(x y) z=(x z) y$. But $(x y) z=$ $z(x y)$ and $(x z) y=(z x) y$ and hence $z(x y)=(z x) y$ and this proves that $A$ is associative.

Now each pre-Lie algebra structure over $g$ is identified with an element of the vector space $\mathfrak{g}^{*} \otimes \mathfrak{g}^{*} \otimes \mathfrak{g}$ and $\boldsymbol{A}(\mathfrak{g})$ is identified with an algebraic subset of $\mathfrak{g}^{*} \otimes \mathfrak{g}^{*} \otimes \mathfrak{g}$. For each $p \in M$, Let $\boldsymbol{A}_{p}$ be the subset of $\boldsymbol{A}(\mathfrak{g})$ consisting of all pre-Lie algebra over $\mathrm{g}$ such that $\mathrm{g}_{p}$ is a left ideal. Then $\boldsymbol{A}_{p}$ is an algebraic subset of $\boldsymbol{A}(\mathrm{g})$ and by Lemma $2, \boldsymbol{A}(M)$ is identified with $\bigcap_{p \in M} \boldsymbol{A}_{p}: \boldsymbol{A}(M)=\bigcap_{p \in M} \boldsymbol{A}_{p}$. Then there exists a finite number of points $p_{1}, \cdots, p_{r}$ in $M$ such that $\boldsymbol{A}(M)=\bigcap_{i=1}^{r} \boldsymbol{A}_{p_{i}}$ and hence $\boldsymbol{A}(M)$ is a complex affine variety. This proves Theorem 2 .

UNIVERSity OF Notre DaMe 


\section{References}

[1] M. Gerstenhaber: The cohomology structure on an associative ring, Ann. of Math. 78 (1963), 267-289.

[2] R.C. Gunning: Special coordinate coverings of Riemann surfaces, Math. Ann. 170 (1967), 67-86.

[3] A. Nijenhuis and R.W. Richardson: Cohomology and deformations in graded Lie algebras, Bull. Amer. Math. Soc. 72 (1966), 1-29. 